\title{
The effect of ion milling on the morphology of ramp-type Josephson junctions
}

\author{
Dave H. A. Blank and Horst Rogalla \\ Low Temperature Division, Department of Applied Physics, University of Twente, P.O. Box 217, \\ 7500 AE Enschede, The Netherlands
}

(Received 21 April 1997; accepted 8 August 1997)

Artificial barriers in Josephson junctions make it possible to change the height and width of the barrier independently. This technique can be realized in high- $T_{c}$ Josephson junctions using the ramp technique. In this article the fabrication of ramp-type junctions is discussed and the importance of the morphology of the ramp is pointed out. Detailed investigations are described which address the surface roughness and the damage due to ion-beam structuring of ramps. It is shown that hard masks can significantly improve the ramp quality by reducing the ion impact angle. Furthermore, annealing of the so structured ramps leads to unit cell steps enforcing a step-flow growth mode.

\section{INTRODUCTION}

The possibility to apply artificial epitaxial barriers makes ramp-type junctions especially attractive as Josephson junctions in high- $T_{c}$ superconducting electronics. ${ }^{1}$ A-b-plane geometry allows for a wide range of coupling strengths between junction electrodes, from direct superconducting coupling over tunneling to insulation, depending on the height and thickness of a homoepitaxial barrier. In contrast, $c$-axis coupling is much weaker and in general Josephson currents cannot be observed. Deposition of layers from the top gives a much higher degree of control, because in that case standard deposition methods can be used and one can easily vary the barrier thickness and material. Ramptype junctions combine in that sense the advantages of a planar design with the strong coupling in the a-b-plane.

Since the first publication on ramp-type junctions, immense progress has been made. The characteristics of the junctions can be tailored by changing the barrier thickness and/or doping the barrier material, e.g., Ga-doping of $\mathrm{PrBa}_{2} \mathrm{Cu}_{3} \mathrm{O}_{7-d}$. However, the electrical characteristic of the ramp-type junction is still not reproducible enough for medium scale integration (more than 100 junctions on one chip). This holds especially true if one compares the junction spread from run to run. One of the reasons is the quality of the ramp, which relies a great deal on an ex-situ etching procedure. Furthermore, the homoepitaxial growth of the barrier material on (etched) ramps is not trivial. With the present fabrication methods a minimum thickness of $8-10 \mathrm{~nm}$ is required to ensure coverage of the whole junction area. ${ }^{2}$ Another aspect is the possible segregation of doped species leading to inhomogeneous electrical properties.

In this contribution we are looking in more detail into the materials aspects of the ramp-type junction. Each process step has been studied extensively to improve the reproducibility and quality of the ramp-type junction. Starting with the etching procedure, we look into the growth process on ramps and the influence of etch damage on this growth.

\section{EXPERIMENTAL}

\section{Junction fabrication}

In the optimization of junction properties and reproducibility of ramp-type junctions, it is necessary to carefully analyze the fabrication process, in order to see which aspects need improvement or adjustment. In Fig. 1 the geometry of a ramp-type junction is given. The fabrication starts with the deposition of the base-electrode and isolation (or buffer) layer. Both should be of high orientational (epitaxy on a single crystal substrate) and structural quality. The critical temperature of the base electrode is important because it determines in combination with the top electrode the maximum operation temperature of the junction. Inhomogeneous superconducting properties throughout the film (caused by impurities or defects) cause irregularities in the barrier-superconductor interface. Surface roughness caused by outgrowths or holes can lead to shorts between the top and base electrodes, thus distorting the Josephson behavior. With ion beam etching, outgrowths or holes leave imprints in the ramp, causing irregularities in the ramp surface.

In the next step, the ramp is structured. This is the most crucial part in the fabrication of ramp-type junctions. Up till now, different approaches have been realized by a number of different groups. Among them:

(i) Shadow mask deposition. ${ }^{3-5}$ In this case the ramp is formed during deposition of the base layers, and no separate step to structure a ramp is necessary. It is, however, very difficult to control the junction area and 


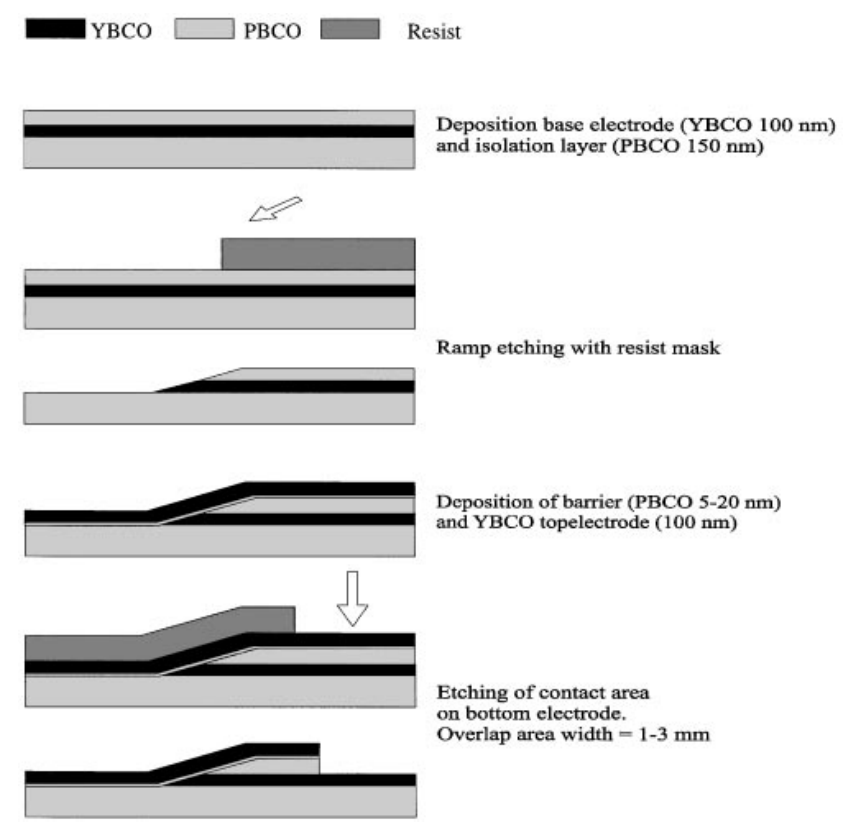

FIG. 1. Schematic procedure of preparation process of ramp-type junctions.

the thickness of the buffer layer. An advantage is that the whole process can be performed in situ.

(ii) Chemical etching. ${ }^{6,7}$ After deposition of a photoresist or $\mathrm{ZrO}$ mask, a ramp is etched using chemical etching with, e.g., Br-ethanol solution. This leads to very small ramp angles $\left(2^{\circ}-3^{\circ}\right)$. The advantage is that the stoichiometry of the surface layer is not changed during etching, provided there is a very pure crystalline base layer. If this is not the case, then the etching rate will not be homogeneous throughout the film and the surface quality will drastically decrease.

(iii) Ion beam etching. ${ }^{1,8-10}$ After deposition of an etch mask, e.g., photoresist or metal, the ramp is etched by an ion beam that strikes the sample at a certain angle. The angle of the ramp has to be well below $45^{\circ}$ to obtain good epitaxial growth of the barrier and the top layer, to prevent tilting of the $c$-axis, and to enable crystal continuity. ${ }^{7}$ To obtain a homogeneous junction area, the angle should not vary too much along the ramp. The main problem in this method is the distortion of the surface layer during the ion bombardment. Furthermore, the sample has to be taken out of vacuum after structuring the ramp to remove the photoresist, exposing the surface to air and humidity.

After structuring the ramp, the barrier and the top electrode are deposited in one run. The ramp surface should provide a good seed layer for the barrier. Surface roughness of the ramp will prevent homogeneous coverage of the ramp by the barrier material and can lead to short circuits. A reduction of the superconducting properties near the interface due to distortions in the ramp surface layer can be caused by the etching process or by diffusion of the barrier material into the superconductor and can lead to a boundary resistance. This resistance between the barrier and the superconductor reduces the superconductive coupling between the electrodes. Such boundary resistance can also be caused by stress due to a lattice mismatch between the barrier and electrode materials. As mentioned before, the thickness and the electrical properties of the barrier (e.g., barrier height) determine the properties of the junction. To be able to grow very thin barriers and avoid a large boundary resistance, the barrier material should have good lattice match to the base layer, and should give a good coverage of the ramp even at very small thickness. Therefore, it should preferably grow in the layer-by-layer mode, or in case of the ramp surface, in step-flow mode.

In a further step, the overlap of the top electrode over the base electrode is (partly) removed by ion beam etching. This overlap (see Fig. 1) forms a parallel plate capacitor parallel to the junction, and thus influences the behavior of the junction. The contribution of this capacitance to the total capacitance of the junction would be quite large (up to $50 \%$ of the total capacitance), depending on the size of the overlap and the dielectric properties of the buffer layer. ${ }^{10}$

Finally, the geometric structure of the junctions (e.g., the widths) and contact paths on the sample are determined.

\section{RESULTS}

\section{A. Ramp-etch procedure}

The morphology of the ramped surface is dependent on the quality of the layer into which the ramp is etched as well as on the actual etching procedure. In this study two different methods for the fabrication of high- $T_{c}$ superconducting thin films were used: pulsed laser deposition (PLD) and off-axis RF-magnetron sputter deposition. As substrates, in both cases $5 \times$ 10-mm [100] $\mathrm{SrTiO}_{3}$ (STO) single crystals were used. For PLD a KrF laser $(248 \mathrm{~nm})$ with an energy density of $15 \mathrm{~mJ} / \mathrm{mm}^{2}$ (spot sizes of $7.5-8.0 \mathrm{~mm}^{2}$ ) and a repetition rate of 2 or $5 \mathrm{~Hz}$ was applied. The oxygen pressure during deposition was $0.15-0.23$ mbar. The deposition rate was $0.1 \mathrm{~nm}$ per pulse, and the substrate was heated to a temperature of $720-780{ }^{\circ} \mathrm{C}$. After deposition, the chamber was filled with oxygen to atmospheric pressure, and the substrate was cooled down to room temperature via the following cooling trajectory: $15 \mathrm{~min}$ at $500{ }^{\circ} \mathrm{C}$ and $15 \mathrm{~min}$ at $400{ }^{\circ} \mathrm{C}$.

In off-axis RF-magnetron sputter deposition we used a mixture of $\mathrm{Ar}$ and $\mathrm{O}_{2}$ (ratio 15:20) with a pressure between 10 and $20 \mathrm{~Pa}$. Traces of $\mathrm{H}_{2} \mathrm{O}$ vapor of about $0.1 \mathrm{~Pa}$ were added in order to stabilize the process. The substrate was heated to $770{ }^{\circ} \mathrm{C}$. Stoichiometric $\mathrm{REBa}_{2} \mathrm{Cu}_{3} \mathrm{O}_{7-d}$ targets were used, mounted on cathodes 
with cylindrical magnetrons. The RF power was $90 \mathrm{~W}$ at 13.56 MHz. The substrate is placed off-axis to prevent resputtering by bombardment with highly energetic ions that are accelerated in a direction normal to the target. The deposition rate in this setup is about $50 \mathrm{~nm} / \mathrm{h}$.

The difference in deposition rate between sputter deposition and PLD leads to differences in the morphology of the thin films. The grain size of sputter deposited films is much larger than that of laser deposited films (400 nm-1 $\mu \mathrm{m}$ and 50-200 nm, respectively). In sputter deposited films the main growth process involved is spiral growth, whereas in PLD films the growth spirals are not that clearly visible, and it resembles more Stranski-Krastanov growth.

The etching of the ramp structure is done by ion beam etching with a $3 \mathrm{~cm}$ ion (Kaufman) source. To obtain only singly ionized Ar, the cathode voltage is kept at $40 \mathrm{~V}$. Typical parameters used are a discharge voltage of $40 \mathrm{~V}$, beam voltages of $50 \mathrm{~V}$ for cleaning of the material (low etch rate), $500 \mathrm{~V}$ for ramp etching, and $1000 \mathrm{~V}$ for etching of sharp metal mask-edges. The accelerator voltage was kept at $100 \mathrm{~V}$ and the beam current at $10 \mathrm{~mA}$. To prevent excessive heating of the sample, the source is pulsed ( $8 \mathrm{~s}$ on, $10 \mathrm{~s}$ off).

The quality and the angle of the ramp is predominantly determined by the properties of the etching mask used. An important factor is the etch rate of the mask (see Fig. 2). As the thickness of the mask decreases during etching, the shadow region of the mask becomes smaller, resulting in a more shallow angle of the $\operatorname{ramp}(\alpha)$ compared to the angle of incidence of the ion beam $(\beta)$.

$$
\begin{aligned}
\alpha & =\arctan \left(\frac{1}{1+x} \tan \beta\right), \\
\text { with } x & =\frac{\text { etching rate mask }}{\text { etching rate film }} .
\end{aligned}
$$

The difference between $\alpha$ and $\beta$ is the impact angle $\gamma$, with which the ion beam strikes the ramp surface area. In Fig. 3 this impact angle is plotted against the

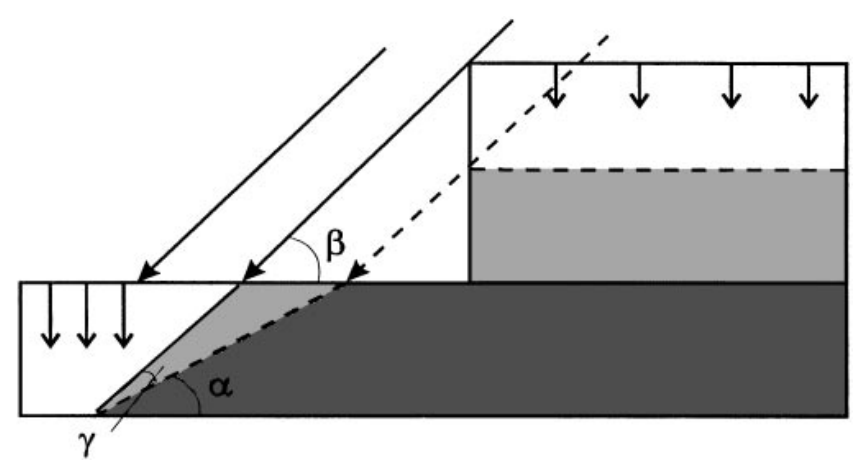

FIG. 2. Influence of the mask etch rate to the ramp angle.

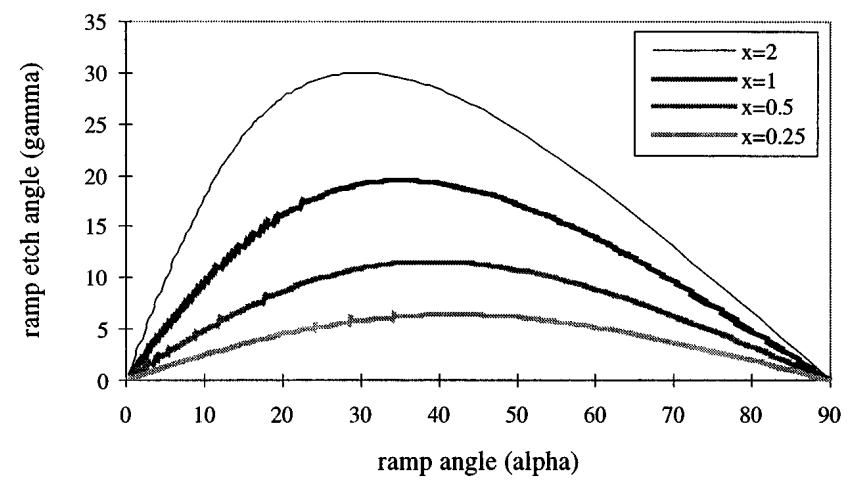

FIG. 3. Impact angle $\gamma$ as a function of the actual ramp for masks with different etching rates, with $x=$ etching rate mask/etching rate film.

ramp angle for masks with different etching rates. If one, for example, considers photoresist, which has an etching rate two times as large as $\mathrm{REBa}_{2} \mathrm{Cu}_{3} \mathrm{O}_{7-d}(x=2), \gamma$ becomes very large, especially in the region of interest: the ramps used in ramp type junctions usually have an angle $\alpha \approx 10-30^{\circ}$. Consequently, fabricating a ramp by using photoresist, the ramp area is etched with an impact angle of $20-30^{\circ}$. This results in a significant etch rate, as shown in Fig. 4, in which the angular dependence of the etching rate is plotted. As can be seen from Fig. 3, the impact angle and thus the surface damage can significantly be reduced by using harder masks.

\section{B. Annealing of ion milled and cleaned ramps}

To verify the effect of the impact angle on the quality of the ramp surface, different samples were prepared by applying a photoresist mask with a grating image aligned with the crystal axis of the $\mathrm{YBa}_{2} \mathrm{Cu}_{3} \mathrm{O}_{7-d}$. The ramps were etched under an angle $\beta$ of $45^{\circ}$.

After etching the ramp and removing the photoresist, the sample was cleaned by the standard etching process. The cleaning improves the interface quality of the ramp significantly, as verified by ramp-type junctions without

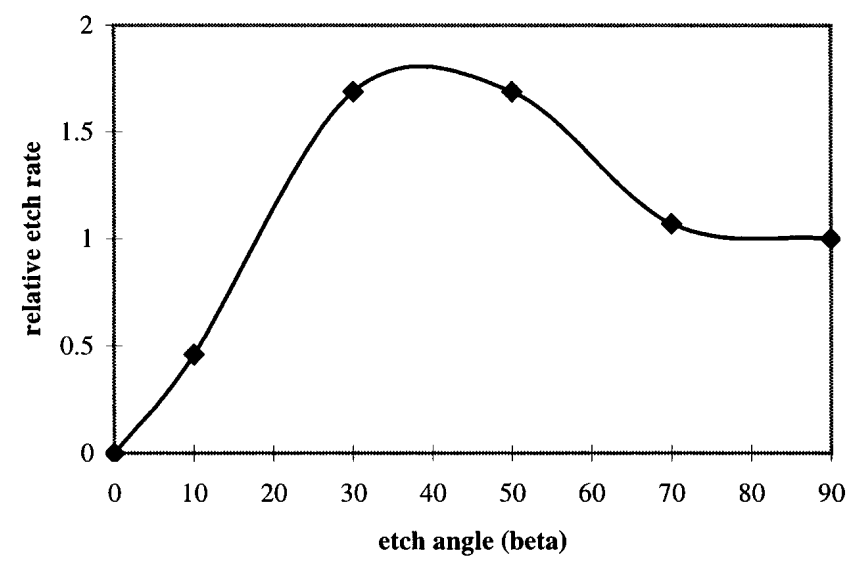

FIG. 4. Dependence of etch rate on angle of incidence for $\mathrm{YBaCuO}$. 
barrier. In this configuration, reduction of the critical current density is caused by interface defects, and is a measure of the total amount of damage that is caused during etching. ${ }^{11}$

To enable reconstruction of the surface layer by enhancing the surface mobility, the freshly etched ramps were annealed at a temperature and oxygen pressure below the stability line in the phase diagram of $\mathrm{YBa}_{2} \mathrm{Cu}_{3} \mathrm{O}_{7-d}{ }^{12}\left(0.1\right.$ mbar oxygen for $1 \mathrm{~h}$ at $\left.800{ }^{\circ} \mathrm{C}\right)$, and subsequently cooled in 200 mbar oxygen to room temperature.

Using fresh and sharp AFM tips, pictures as shown in Fig. 5 could be observed. In this figure the deflection data (derivative of the height or error-signal mode) of the AFM image is given. This deflection mode provides a sensitive edge detection technique. Small structures are visible on top of the whole film, leading to an overall increase of the roughness. Typical sizes of these features are about $20-40 \mathrm{~nm}$ in width and about $10 \mathrm{~nm}$ high. They originate from the damaged surface layer during annealing, or degradation due to contact with air. In order to remove amorphous material, a chemical etching procedure $(10 \mathrm{~s}$ in ethylenediaminetetraacetic acid, EDTA) after the ion milling and prior to the anneal and crystallization step was applied. However, it did not reveal a clear difference in the AFM data. Again features were visible over the whole treated area; see Fig. 6 . This indicates that the etching procedure does not lead to an amorphization of the surface.

In order to further investigate the effect of the ion milling procedure on the surface morphology, samples

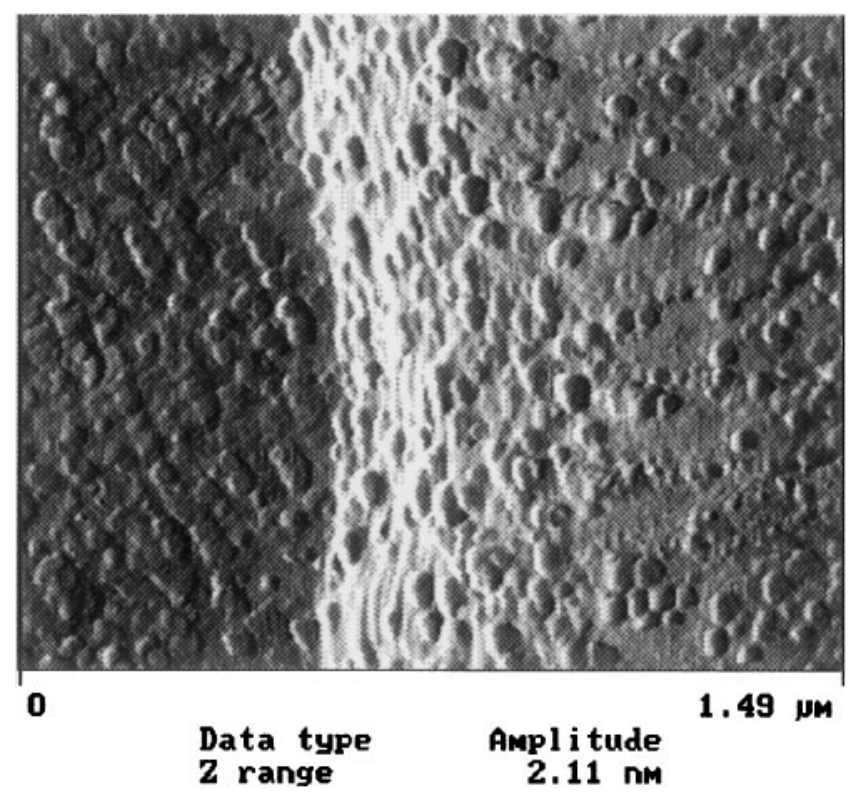

FIG. 5. Deflection AFM micrograph of a ramp surface of $\mathrm{YBaCuO}$ after etching, cleaning, and annealing for $1 \mathrm{~h}$ at $800{ }^{\circ} \mathrm{C}$ and $0.1 \mathrm{mbar}$ oxygen pressure. were directly annealed for $1 \mathrm{~h}$ at $700{ }^{\circ} \mathrm{C}$ and $0.1 \mathrm{mbar}$ $\mathrm{O}_{2}$ and compared with samples treated with the same annealing procedure after a cleaning by 2 pulses of $500 \mathrm{eV}$ and 10 pulses of $50 \mathrm{eV}$ of $\mathrm{Ar}^{+}$ions. The result is shown in Fig. 7. The left part shows the result after annealing only: the film looks exactly like a fresh film, whereas on the right part (with a cleaning step) the damage is clearly visible. In fact, AFM data directly after ion milling (without anneal step) already showed the increase in roughness. There was no difference between films made with sputter deposition and PLD.

The nature of the features that appear on the surface after etching is unknown. The features resemble structures that are reported in the literature to be $\mathrm{Y}_{2} \mathrm{O}_{3}$ grains. ${ }^{13}$ With scanning Auger we observed in the etched surface a reduction of the $\mathrm{Cu}$ content of about $60 \%$.

The main conclusion from the experiments on ion milling of ramps and thin films is that the surface of $\mathrm{REBa}_{2} \mathrm{Cu}_{3} \mathrm{O}_{7-d}$ is severely damaged by the etching procedure. This damage is visible as a modification of the surface, leading to a disordered and rough surface. No relation is seen between the energy of the sputter ions and the appearance of the damage. As far as junction fabrication is concerned, this can seriously hamper the growth of thin barriers. Minimization of $\gamma$, which can be done by using hard etching masks, reduces the ion bombardment and leads to less damage at the ramp surface.

\section{Hard etching masks}

Applying hard etching masks (e.g., TiN), ramps were structured into pulsed laser deposited $\mathrm{YBa}_{2} \mathrm{Cu}_{3} \mathrm{O}_{7-d}$ thin films. The angle of incidence was $30^{\circ}$, resulting in a ramp angle of about $20^{\circ}$. The uniformity of the ramp angle and surface roughness of the ramp improved significantly. In Fig. 8 an AFM micrograph of the ramp, including a zoom into the ramp area, is given. The ramp is very well defined: there is no concavity visible in the lower region of the ramp. The ramp area is completely smooth.

The surface layer of this ramp is not very likely to enhance the desired step-flow growth of the barrier, since no steps are visible. Therefore, annealing experiments were conducted on these ramps in order to force the surface to recrystallize. This was done by annealing the sample slightly below the decomposition line in the phase diagram $\left(0.1\right.$ mbar oxygen pressure, $780{ }^{\circ} \mathrm{C}$, for $1 \mathrm{~h}$, subsequently cooled to $450{ }^{\circ} \mathrm{C}$, and then cooled to room temperature in 200 mbar oxygen pressure). The result is given in Fig. 9. On quite a large area of the ramp faceting is visible, showing steps of unit-cell height. In this example the ramp is not aligned with the crystal axis, which explains the short facets. In the experiments of Verhoeven, a relation was found between alignment of the ramp and the length of the facets in sputtered films. ${ }^{11}$ 

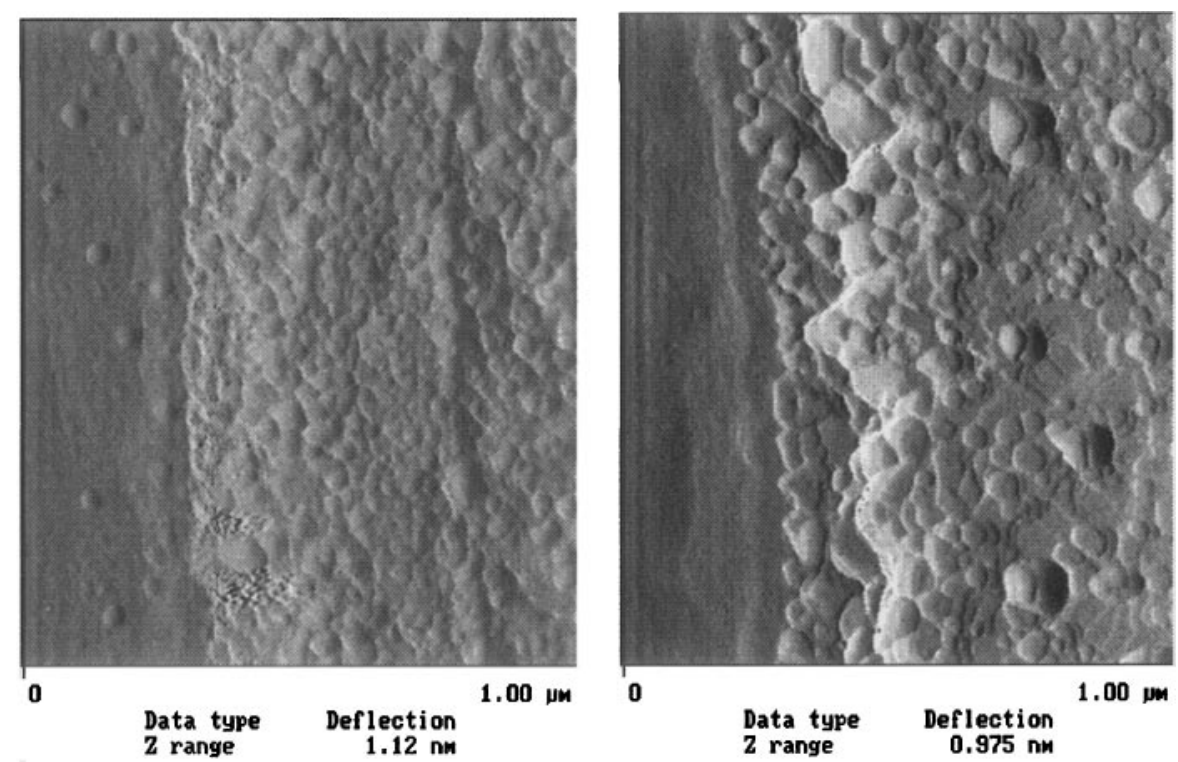

FIG. 6. AFM micrographs of a chemically cleaned ramp after a subsequent $50 \mathrm{eV} \mathrm{Ar}$ ion clean (left) and the same ramp after extra cleaning step and annealing (right).
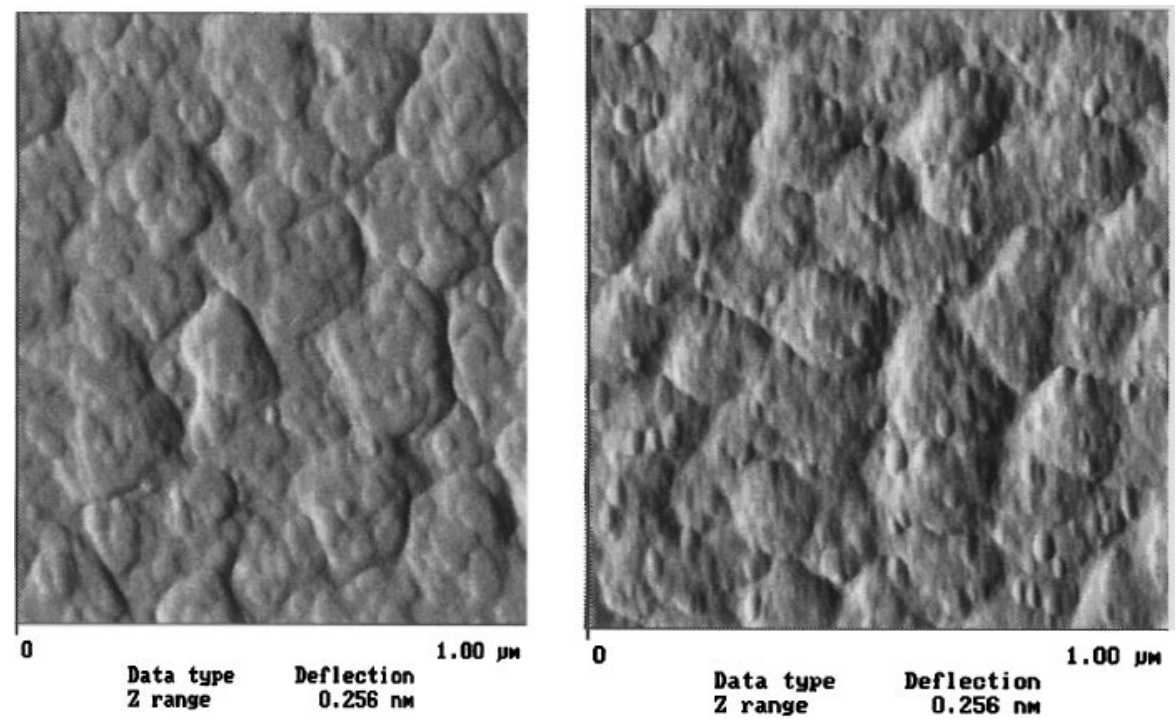

FIG. 7. Deflection pictures of $\mathrm{YBCO}$ film after annealing (left) and after annealing with preceding $\mathrm{Ar}^{+}$cleaning (right).

It is expected that the facets in pulsed laser deposited films will be smaller, because the grain size of PLD films is smaller.

These steps of unit cell height are also an indication that the crystallinity extends all the way to the ramp surface. There is no "damage region" present. Consequently, this process leads to clean interfaces. Indeed, Josephson junctions made with the hard mask technique show lower $R_{n}$-values compared to junction with identical barrier thickness, made by the standard (photoresist mask) technique. ${ }^{11}$ Typical values of the $I_{c} R_{n}$ product found in Verhoeven's work, for junctions made with a $10 \%$ Ga-doped PrBCO barrier and a thickness of $10 \mathrm{~nm}$, are $1.0 \mathrm{mV}$ at $7 \mathrm{~K}$. Here, we obtained for the same barrier material, thickness and temperature typical $I_{c} R_{n}$ products of $0.8 \mathrm{mV}$.

\section{CONCLUSIONS}

Ion milling of the $\mathrm{REBa}_{2} \mathrm{Cu}_{3} \mathrm{O}_{7-d}$ surface leads to changes in surface morphology, leading to the appearance of irregularities, as revealed by AFM measurements. These irregularities are more pronounced after recrystallization of the surface by annealing just below the decomposition line of the $\mathrm{REBa}_{2} \mathrm{Cu}_{3} \mathrm{O}_{7-d}$. These irregularities are also present on the ramp surface, which 

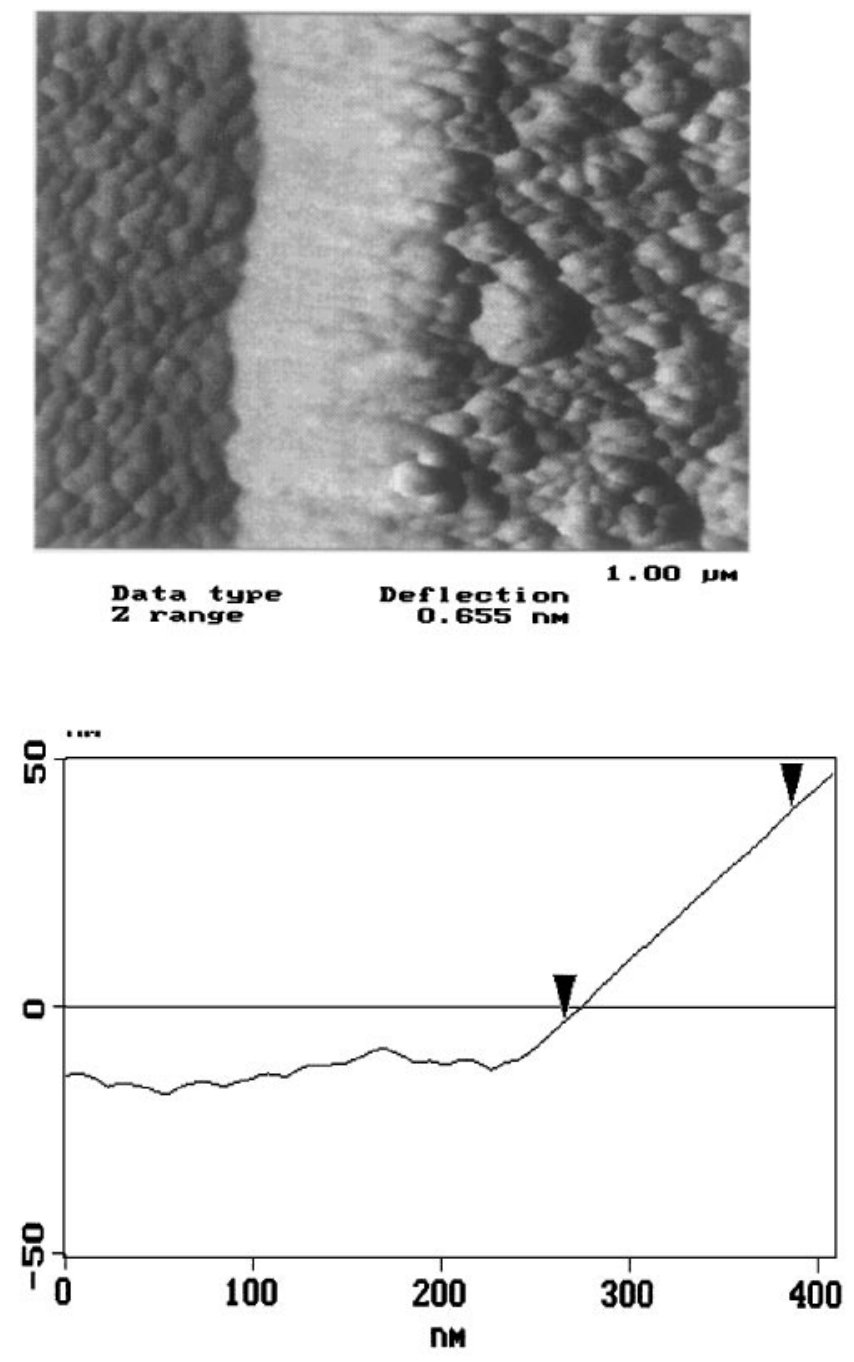

FIG. 8. Deflection picture of ramp in YBCO, and cross section of zoom on lower part of the ramp.

have been made using a soft mask, due to the relatively large impact etch angle. Using a hard mask, like TiN, this impact etch angle decreases significantly, leading to a smoother and more homogeneous ramp surface.

The ramp made by the TiN mask shows, in contrast with a photoresist mask, no concavity in the lower region of the ramp. The ramp surface is found to be extremely smooth, and no structures are visible. After recrystallization of the surface by annealing, these ramps show unit cell steps, having the ideal properties for barrier growth. Junctions made with the TiN mask show a lower normal resistance compared to junctions with an identical barrier made with a soft mask, indicating cleaner interfaces.

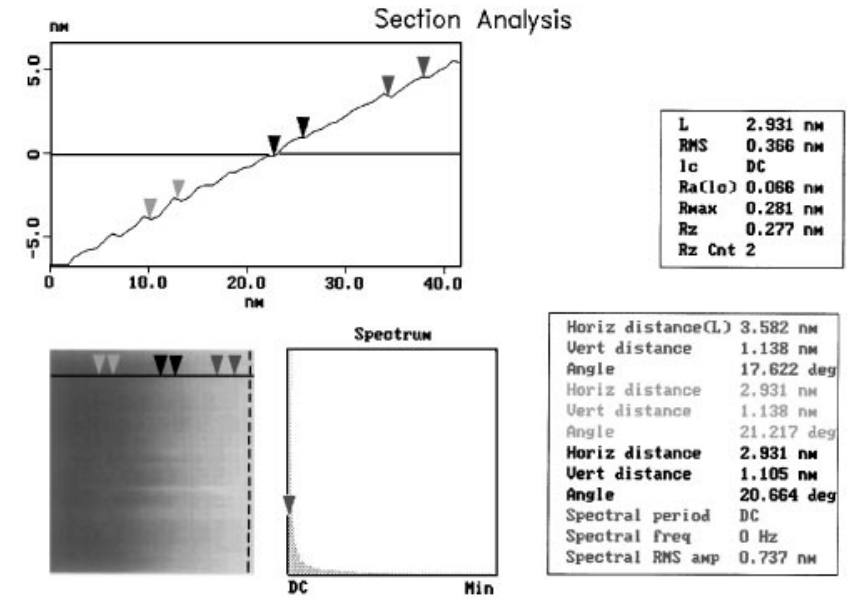

FIG. 9. Cross section of zoom on annealed ramp made with TiN mask showing unit cell steps.

\section{ACKNOWLEDGMENTS}

The authors would like to acknowledge Guus Rijnders, Roger Bergs, and Frank Roesthuis for their help in the experimental study and useful discussions.

\section{REFERENCES}

1. J. Gao, W. A. M. Aarnink, G.J. Gerritsma, and H. Rogalla, Physica C 171, 126 (1990).

2. M. A. J. Verhoeven, R. Moerman, M.E. Bijlsma, A. J.H.M. Rijnders, D. H. A. Blank, G. J. Gerritsma, and H. Rogalla, Appl. Phys. Lett. 68, 1276 (1996).

3. D. K. Chin and T. van Duzer, Appl. Phys. Lett. 58, 753 (1991).

4. Th. Becherer, C. Stölzel, G. Adrian, and H. Adrian, Phys. Rev. B 47, 14650 (1993).

5. C. Stölzel, M. Siegel, G. Adrian, C. Krimmer, J. Söllner, W. Wilkens, G. Schulz, and H. Adrian, Appl. Phys. Lett. 63, 2970 (1993).

6. M.I. Faley, U. Poppe, H. Soltner, C.L. Jia, M. Siegel, and K. Urban, Appl. Phys. Lett. 63, 2138 (1993).

7. R. Dömel, C. L. Jia, C. Copetti, G. Ockenfuss, and A. I. Braginski, Supercond. Sci. Technol. 7, 277 (1994).

8. C. L. Jia, B. Kabius, K. Urban, K. Herrman, G. J. Cui, J. Schubert, W. Zander, A. I. Braginski, and C. Heiden, Physica C 175, 545 (1991).

9. B. D. Hunt, M.C. Foote, and L. J. Bajuk, Appl. Phys. Lett. 59, 982 (1991).

10. J. B. Barner, B.D. Hunt, M.C. Foote, W. T. Pike, and R.P. Vasquez, Physica C 207, 381 (1993).

11. See, e.g., M. A. J. Verhoeven, High- $T_{c}$ superconducting ramp-type junctions, Ph.D. Thesis, University of Twente, ISBN 90-365-08177 (1996), M. A. J. Verhoeven, G. J. Gerritsma, H. Rogalla, and A. A. Golubov, Appl. Phys. Lett. 69, 848 (1996).

12. R. H. Hammond and R. Bormann, Physica C 162-164, 703 (1989).

13. A. Catana, J. G. Bednorz, Ch. Gerber, J. Mannhart, and D. G. Schlom, Appl. Phys. Lett. 63, 553 (1993). 